\title{
Demineralization Enables Reeling of Wild Silkmoth Cocoons
}

\author{
Tom Gheysens, ${ }^{*,+}$ Andrew Collins, ${ }^{\ddagger}$ Suresh Raina, ${ }^{\S}$ Fritz Vollrath, ${ }^{*,+}$ and David P. Knight ${ }^{\dagger}$ \\ ${ }^{\dagger}$ Department of Zoology, University of Oxford, South Parks Road, Oxford, OX1 3PS, United Kingdom \\ ${ }^{\ddagger}$ Centre for Organized Matter Chemistry, University of Bristol, School of Chemistry, Bristol, BS8 1TS, United Kingdom \\ ${ }^{\S}$ Commercial Insects Program, International Centre of Insect Physiology and Ecology, Nairobi, Box 30772, 00100, Kenya
}

\begin{abstract}
Wild Silkmoth cocoons are difficult or impossible to reel under conditions that work well for cocoons of the Mulberry silkmoth, Bombyx mori. Here we report evidence that this is caused by mineral reinforcement of Wild Silkmoth cocoons and that washing these minerals out allows for the reeling of commercial lengths of good quality fibers with implications for the development of the "Wild Silk" industry. We show that in the Lasiocampid silkmoth Gonometa postica, the mineral is whewellite (calcium oxalate monohydrate).

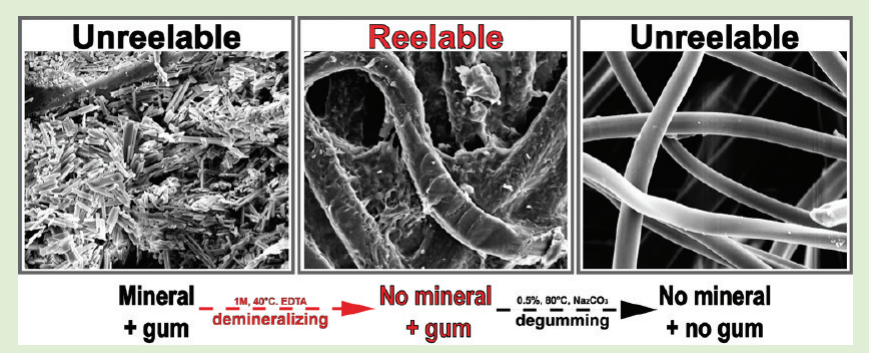
Evidence is presented that its selective removal by ethylenediaminetetraacetic acid (EDTA) leaves the gum substantially intact, preventing collapse and entanglement of the network of fibroin brins, enabling wet reeling. Therefore, this method clearly differs from the standard "degumming" and should be referred to as "demineralizing". Mechanical testing shows that such preparation results in reeled silks with markedly improved breaking load and extension to break by avoiding the damage produced by the rather harsh degumming, carding, or dry reeling methods currently in use, what may be important for the development of the silk industries not only in Asia but also in Africa and South America.
\end{abstract}

\section{INTRODUCTION}

Cocoons of the Mulberry silkworm Bombyx mori are relatively easy to soften and reel in comparison with most other commercially attractive silkmoths. ${ }^{1}$ Indeed, perhaps this feature has led to the predominance of $B$. mori among natural silks or alternatively it is the outcome of 6000 years of selection by man for a silk with high commercial potential. After all, softening and reeling that are both easy to administer and gentle on the fiber are key to the material and its usability. ${ }^{2}$

Typically, B. mori cocoons are softened by submersion in hot water. This treatment swells and partly dissolves the sericin gum, which coats and cements the fibroin filaments (brins) together in the cocoon. In contrast to the cocoons of B. mori, the cocoons of most other (traditionally called "Wild") silkmoth species are often heavily mineralized with calcium oxalate. ${ }^{3-6}$ Moreover, sometimes such "Wild" cocoons are additionally stabilized by oxidative phenolic tanning, dityrosine cross-linking, and tannins derived from the caterpillar's food plant. ${ }^{7,8}$ Mineralization is probably more important than the tanning in making Wild Silk cocoons difficult to soften and reel.

Traditionally, two degumming approaches have been attempted to overcome this set of problems. The cocoons of Saturnid Wild Silkmoths can generally be softened and degummed by boiling in concentrated sodium carbonate solutions for hours with or without the addition of soap. ${ }^{9}$ However even with this relatively harsh treatment the wet reeling method used for Mulberry silk is not possible, necessitating the use of greater force obtained by dry or semidry reeling. ${ }^{10}$ The cocoons of several non-Saturnid Wild Silkworms, particularly those of Gonometa spp. are even more resistant to softening and require prolonged retting or treatment with pineapple extracts containing bromelain to remove the sericin. ${ }^{11}$ Even with this treatment they still cannot be reeled and must be carded to be spun into a short staple yarn. Both these degumming methods tend to damage the silk fibers with the latter approach especially producing much weakened fibers capable only of short staple lengths that can only be spun into weak yarns of irregular thickness and hence considered of poor quality.

The marked contrast in the methods of obtaining silk from the cocoons of Wild Silkmoths with those used for the highly domesticated B. mori suggests a fundamental difference in cocoon composition. We formulated the hypothesis that the principal difference lay in the mineralization that is so common in Wild Silk moths yet absent in B. mori and that this difference arises from the cementing together of the silk fibers in a mineralized matrix in Wild Silk cocoons making them impossible to wet reel. Here we first report our tests of this hypothesis by examining the effect of demineralizing a range of Wild cocoons on their ability to be wet reeled. Then we focus on the Lasiocampid Gonometa postica because it makes an exceptionally tough mineralized cocoon containing a very interesting silk with commercial potential. Here we characterize in detail the constituent mineral added to the

Received: March 14, 2011

Revised: April 8, 2011 
sericin gum and the effect of its selective removal on reeling and on the mechanical properties of the resulting silk filaments. Finally we compare the effect of the novel demineralizing method described here with that of the currently used method, pineapple juice digestion and carding, on the mechanical properties of the resulting fibers.

\section{MATERIALS AND METHODS}

Materials. Emerged cocoons of the Saturnid family, Attacus atlas, Antheraea frithi, Antheraea mylitta, Antheraea pernyi, Antheraea polyphemus, Antheraea roylei, Antheraea yamamai, Bunaea alcinoe, Callosamia promethean, Cricula andrei, Hyalophora cecropia, Hyalophora gloveri, Philosamia canninggii, Philosamia cynthia advena, Saturnia pavonia, and Saturnia pyri, and from the Bombycid family, Bombyx mori, were bought from Worldwide Butterflies (U.K.) where they had been bred. Cocoons from the Lasiocampid Gonometa postica were either obtained from old collections or, for a more detailed investigation, from ICIPE in Kenya who obtained them fresh from a silk growers cooperative in Mwingi. For the comparison of silk fibers reeled using the methods described here with traditionally carded fibers, lengths of spun yarn from G. postica was also supplied by ICIPE having been obtained from the same batch of cocoons supplied to us. This yarn had been prepared by degumming the cocoons in a standardized protocol using a pineapple extract degumming, hand carding cocoons, and hand spinning.

For studying the removal of mineral, several samples were used from each type of cocoon, typically after the emergence of the moth, and the pupal remains were removed through the emergence hole using forceps. From each cocoon, 15 discs with a diameter of $4.86 \mathrm{~mm}$ were pressed out of the cocoon at random locations with a sharp hole punch. They were then subjected to demineralization with or without degumming as described below. Untreated samples were used as a control. The thickness of the discs was measured before and after treatments using a digital micrometer (Mitutoyo, IP 65) taking care not to compress the disk. During treatment, the discs and sample number engraved with a laser on a rectangular piece of plastic (polystyrene) were enclosed in small fine mesh nylon bags made with a bag sealer.

Furthermore, we tested the effects of the generic treatments described below in considerable detail on one specific Wild Silk. For this we chose Gonometa postica, as this species (a) has been the subject of an excellent study of its ecology and behavior ${ }^{12}$ and (b) has the most heavily mineralized cocoon of the 17 Wild moths studied (see Figure 3), as well as (c) producing a silk that seems to have considerable commercial potential, while at the same time having had the least commercial exploitation so far, due to the inability to reel silks of a consistently high quality. All together these features made G. postica the most challenging of the Wild Silks to reel. In order to avoid breaks in the fiber before reeling, G. postica cocoons were harvested before emergence of the moth, while still attached to pieces of twig, and were also checked for drill-holes produced by parasitic wasps. All cocoons were stored at $-25{ }^{\circ} \mathrm{C}$ for about a month prior to use. For the detailed identification of the mineral, four cocoons of G. postica were ground in a mortar and the resulting white powder was analyzed.

Methods. Treatments. Demineralizing Method. Many agents including aqueous solutions of ethylenediamine-tetraacetic acid (EDTA), citric acid (lemon juice, orange juice), formic acid, aluminum nitrate, and phosphate ions are known to remove calcium oxalate by chelating calcium ions or by directly dissolving it. Pilot experiments showed that of these reagents, warm concentrated aqueous solutions of EDTA at a $\mathrm{pH} \geq 7$ was the fastest at removing calcium oxalate from cocoons; a decrease in temperature, EDTA concentration or $\mathrm{pH}$ markedly increased demineralization time (unpublished results). Accordingly we used a standardized protocol for demineralization as follows. An aqueous $1 \mathrm{M}$ solution of ethylenediaminetetraacetic acid (EDTA) was adjusted to $\mathrm{pH} 10$ with saturated sodium hydroxide solution. The samples ( 1 cocoon or 10 discs per $600 \mathrm{~mL}$ ) were immersed in this solution for $72 \mathrm{~h}$ at $40^{\circ} \mathrm{C}$ and gently stirred. The cocoons and discs were kept under the surface of the solution by means of a plastic mesh, using a partial vacuum to encourage penetration of the solution. The cocoons were then thoroughly washed with running tap water (three times $3 \mathrm{~h}$ ) and subsequently reeled in tap water at room temperature using the standard method for B. mori silk. The cocoon discs were washed in the same way and air-dried overnight at room temperature. The method described here forms the basis of a patent application (GB 1018269.9).

Degumming Method. Half of the reeled fibers (demineralized) and five demineralized discs of each cocoon were degummed following the industrial standard method for B. mori skeins; a $0.5 \%$ sodium carbonate solution $\left(\mathrm{Na}_{2} \mathrm{CO}_{3}\right)$ at $80{ }^{\circ} \mathrm{C}$ for $1 \mathrm{~h}$ under stirring. Afterwards, the samples were thoroughly washed (three times) with deionized water $(5 \mathrm{~L})$ for $2 \mathrm{~h}$ and air-dried overnight at room temperature.

Analysis of Mineral Removal. Thermal Gravimetric Analysis (TGA). The samples were loaded into a Q500 TGA (TA Instruments) and were equilibrated for $30 \mathrm{~min}$ isothermally at $26^{\circ} \mathrm{C}$ and a sample purge flow of $60 \mathrm{~mL} / \mathrm{min}$ with nitrogen (balance purge flow $40 \mathrm{~mL}$ / $\mathrm{min}$ ), the latter to ensure the weight change was due to mineral/gum removal and not due to water loss/gain of the sample dependent on environmental conditions. A total of 10 samples per cocoon $(n=5)$ before and after demineralizing were weighed, and the final weights were averaged per cocoon.

Scanning Electron Microscopy (SEM). Natural, demineralized, and combined demineralized and degummed fibers and discs were sputter coated using an AuPd target for $180 \mathrm{~s}$ resulting in a 6-10 nm layer and imaged using a Jeol Neoscope, JCM-5000 (Nikon, UK) under high vacuum at $15 \mathrm{KeV}$.

Energy-Dispersive X-ray Spectroscopy (EDX). A JEOL JSM 6330 operating with an an ISIS 300 EDX unit (Oxford Instruments) with a working distance of $15 \mathrm{~mm}$ and beam energy of $20 \mathrm{Kv}$ was used. Samples were sputter coated in $15 \mathrm{~nm}$ of gold prior to analysis.

$X$-ray Diffractometry (XRD). A Bruker D8 Advance powder diffractometer, with $2 \theta$ from 5 to $50^{\circ}$ in step increments of $0.01967^{\circ} / \mathrm{s}$ was used.

For EDX and XRD, the heavily mineralized outer part of one disk per cocoon $(n=5)$ was used before and after demineralizing. Representative spectra for each instrument and treatment are shown below.

Fourier Transform Infrared Spectroscopy (FTIR). A Nicolet 6700 Fourier transform infrared spectrometer (FTIR) equipped with a liquid nitrogen cooled MCT-A detector was used with a bounce diamond attenuated total reflectance (ATR) sampling accessory (Thermo Electron Corp., Madison, WI). Spectra were acquired at $4 \mathrm{~cm}^{-1}$ resolution from 7000 to $400 \mathrm{~cm}^{-1}$ using a Happ-Genzel apodization, a Metz phase correction, and no zero filling. Spectra were obtained from the average of 32 scans at a $5.0632 \mathrm{~cm} / \mathrm{s}$ mirror speed and normalized for their intensity. All spectral operations were performed using Omnic 7.3 (Thermo Scientific, Madison, WI). Five discs of each cocoon $(n=5)$ were sampled three times on each side (inside and outside of the disk) before and after demineralizing. All spectra per treatment and disk side were averaged. To obtain an estimate of mineralization of the different cocoon shells from the FTIR spectra, the ratio was calculated of the absorbance of the oxalate peak at $\sim 1312 \mathrm{~cm}^{-1}$ to the absorbance of the amide II region from 1590 to $1504 \mathrm{~cm}^{-1}$.

$$
\text { ratio }_{\text {cocoonshell }}=\left(\frac{A_{1312 \mathrm{~cm}^{-1}}}{A_{1590-1540 \mathrm{~cm}^{-1}}}\right)
$$

The ratio was calculated from spectra taken from the outer surface of cocoon shells $(n=5)$ of the different species. For comparison the same was done for calcium oxalate hydrate (Sigma) as a reference standard for maximum mineral coverage. For B. mori, which has no 
calcium oxalate, and for pure calcium oxalate hydrate, which has no amide II band, the absorbance at this wavenumber was taken for the calculation. This ratio gives an estimated approximate value which depended on variables that could not be strictly standardized including the position of the sample site in the cocoon, the extent of compaction in the cocoon as received and the oxalate content of the caterpillar's food plant.

Reeling. Cocoons were wet reeled in water at room temperature with a reeling speed of $103 \mathrm{~mm} / \mathrm{s}$. The length of reeled silk and the number of breaks during reeling were analyzed from recordings made by a Pluscom Camera (Vimicro301 Neptune).

Tensile Testing. Samples for tensile testing were prepared from the demineralized $(n=25)$ and from demineralized/degummed $(n=25)$ reeled fibres of four cocoons. Samples from the commercial G. postica yarn were obtained by gently teasing out brins $(n=30)$. In all cases, single brins were mounted without preload onto cardboard frames with an initial gauge length of $15 \mathrm{~mm}$. They were then equilibrated at $40 \% \mathrm{RH}$ and $26^{\circ} \mathrm{C}$ for $5 \mathrm{~min}$ prior to tensile testing under the same conditions on an Instron Universal Materials Testing Instrument at a strain rate of 50\% per minute using a $5 \mathrm{~N}$ load cell. Cross sectional areas of the fibres were prepared by clamping orientated silk threads in a holder and cutting them across with a new razor blade before sputtering them with gold and viewing them in the SEM. Digital images of transverse sections on SEM micrographs were manually circled and analyzed with ImageJ 1.42q. Normalized cross-sectional areas were obtained by averaging 150 brins for each sample. The tensile parameters were calculated with a home designed program in Excel (Tensile Import v2.0). The J-integral method was used to determine the energy to break. Stress strain curves were plotted using Origin Pro 8. Exlstat 7.5.2 was used for statistical analysis of the data with ANOVA (two tailed).

\section{RESULTS}

SEM Observation of the Mineral and Its Removal. In general, the outer surface of the cocoons in all species studied was covered with a granular material (see Figure 1 (left-hand side) and Figure 2a,b). EDX and FTIR when taken together indicated that this granular material consisted predominantly of calcium oxalate (spectra were similar to Figure 4c, 3). The cubic crystals observed in Figure $1 \mathrm{~h}$ and the raphides in Figure $2 \mathrm{~b}$ are likely different crystal forms of calcium oxalate. Ranking the different species according to their estimated degree of mineralization (see Figure 3) immediately revealed the two extremes; B. mori, with no calcium oxalate present in the cocoon shell, and G. postica, with calcium oxalate covering the entire cocoon shell with no visible silk. There appeared by eye to be a close relationship between the estimated degree of mineralization and the SEM appearance of the outermost part of the cocoon shell. In this connection, it is interesting to note that the Wild Silks that are commercially exploited at present, like A. pernyi and $A$. mylitta, are fairly low in their degree of mineralization (ratio $\sim 1$, Figure 3 ). This granular mineral coating filled the interstices between fibers. In the species studied, this resulted in a compact and hard cocoon shell impossible to wet reel in all species except B. mori (see Figure 3).

After demineralizing, large quantities of granular material were no longer seen on the surfaces of, and interstices between the fibers in all species, exposing the underlying network of silk fibers in the cocoon shell (see Figure 1 (right-hand side)). EDX and FTIR showed that calcium oxalate was removed after demineralizing the cocoon shells (spectra were similar to Figure 4a,b, and c, (red trace)). All demineralized cocoon shells were still intact and similar in appearance to Figure 2c.
In more detail, all G. postica cocoon discs lost about $24 \%$ of their original weight after the demineralizing treatment $(p<0.001$; see Table 1$)$. Also, the measured mean relative density significantly decreased (from $0.541 \mathrm{~g} / \mathrm{cm}^{3}$ to $0.474 \mathrm{mg} / \mathrm{cm}^{3}$; $p<0.0001$ ) after demineralizing. Figure 2a,b shows that the outer surfaces of the natural cocoons of G. postica were heavily mineralized, with a complete covering of narrow elongated monoclinic crystals similar in size and shape to the calcium oxalate crystals observed in Saturnid cocoons described elsewhere. ${ }^{3-6}$ The cocoon fibers were difficult to distinguish (Figure $2 b$ (arrows)) and the cocoon appeared very compact.

After demineralizing the cocoon discs, all mineral crystals had disappeared and the underlying fibrous structure became visible (see Figure 2c,d). A network of cocoon fibers was now visible and the cocoon appeared less compact with many cavities and holes left by removing the mineral. In more detail it was clear that the surrounding gum was still intact, connecting all cocoon fibers together in a bonded but open network. The continuing presence of the gum after demineralization was further confirmed by the observation that the places where the crystals had been incorporated in the gum were still visible as imprints in the gum coating (see Figure 2d, arrows). When degumming the cocoons directly from the natural cocoon or after first demineralizing, the compact network and binding between cocoon fibers broke down completely (see Figure 2e,f) and the cocoon disk drastically expanded in volume, as shown by a decrease in density, to give a tangled loose mesh of fibers. In Figure $2 \mathrm{f}$, the fibers after degumming appeared very smooth and no crystal imprints were found confirming the removal of gum. On the surface of some smooth fibers, although the original bonded network had broken down, the points at which the fiber had been in contact with other fibers was still recognizable as faint deformations in the surface of the fibroin of the brins (see Figure 2f, arrows).

Spectrographic Evidence for the Identification of the Mineral and Its Removal. Figure 4 shows spectrographic evidence from EDX, XRD, and ATR-FTIR for the identification of the mineral on/in the cocoon and for the effectiveness of its removal by demineralization with EDTA. The extracted powder from the cocoons of $G$. postica clearly showed the presence of calcium, oxygen and carbon in the EDX (Figure 4a, blue trace). The EDX of the cocoon discs also indicated that, after demineralizing, the calcium and oxygen peaks that were initially present in the untreated sample (see Figure 4a, black trace) were no longer present and the carbon peak had decreased (see Figure 4a, red trace).

The XRD spectra of the powder (Figure $4 \mathrm{~b}$, blue) showed major reflections at $2 \theta[100],[020],[040],[122],[200]$, and [123] that could be indexed to calcium oxalate hydrate (JCPDS card of whewellite, pattern 01-077-1160). Further, XRD demonstrated that the six peaks characteristic for calcium oxalate hydrate in the natural sample (Figure 4b, black) were lost after demineralizing the cocoon discs (Figure 4b, red). The FTIRspectra of the extracted powder and the outside of the mineralized cocoon discs (Figure 4c, 2 and 3) matched the spectrum of pure calcium oxalate hydrate (Sigma; Figure 4c, 1) and confirmed that there was a large quantity of calcium oxalate on the outside of the cocoon discs. On the inside of the undemineralized cocoon discs, some calcium oxalate peaks could be seen superimposed on the silk spectrum, but at much reduced height (Figure 4c, 4). After demineralizing the cocoon discs, the peaks characteristic for calcium oxalate (main peaks at 1599.8 and $1311.8 \mathrm{~cm}^{-1}$ ) disappeared completely on the outside as well as on the inside of the 

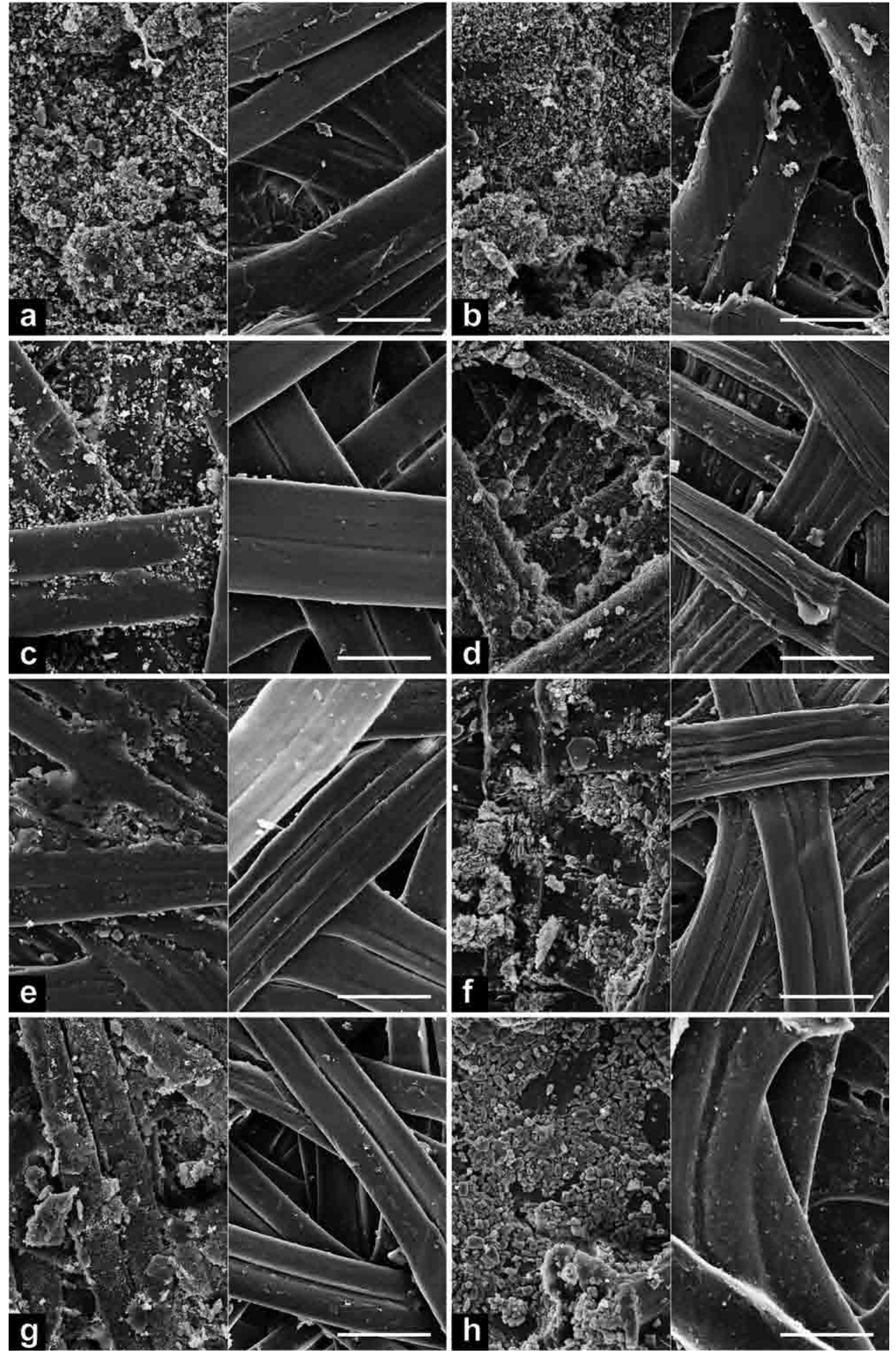

Figure 1. High magnification pairs of SEM pictures $(500 \times$, scale bar $50 \mu \mathrm{m})$ of the same untreated (mineralized; left-hand side) and treated (demineralized; right-hand side) cocoon shell for some selected Silkmoth species: Antheraea frithi (a), Antheraea mylitta (b), Antheraea pernyi (c), Attacus atlas (d), Bunaea alcinoe (e), Hyalophora cecropia (f), Philosamia canninggii (g), Saturnia pyri (h). All other Wild Silk species studied exhibited similar results. 

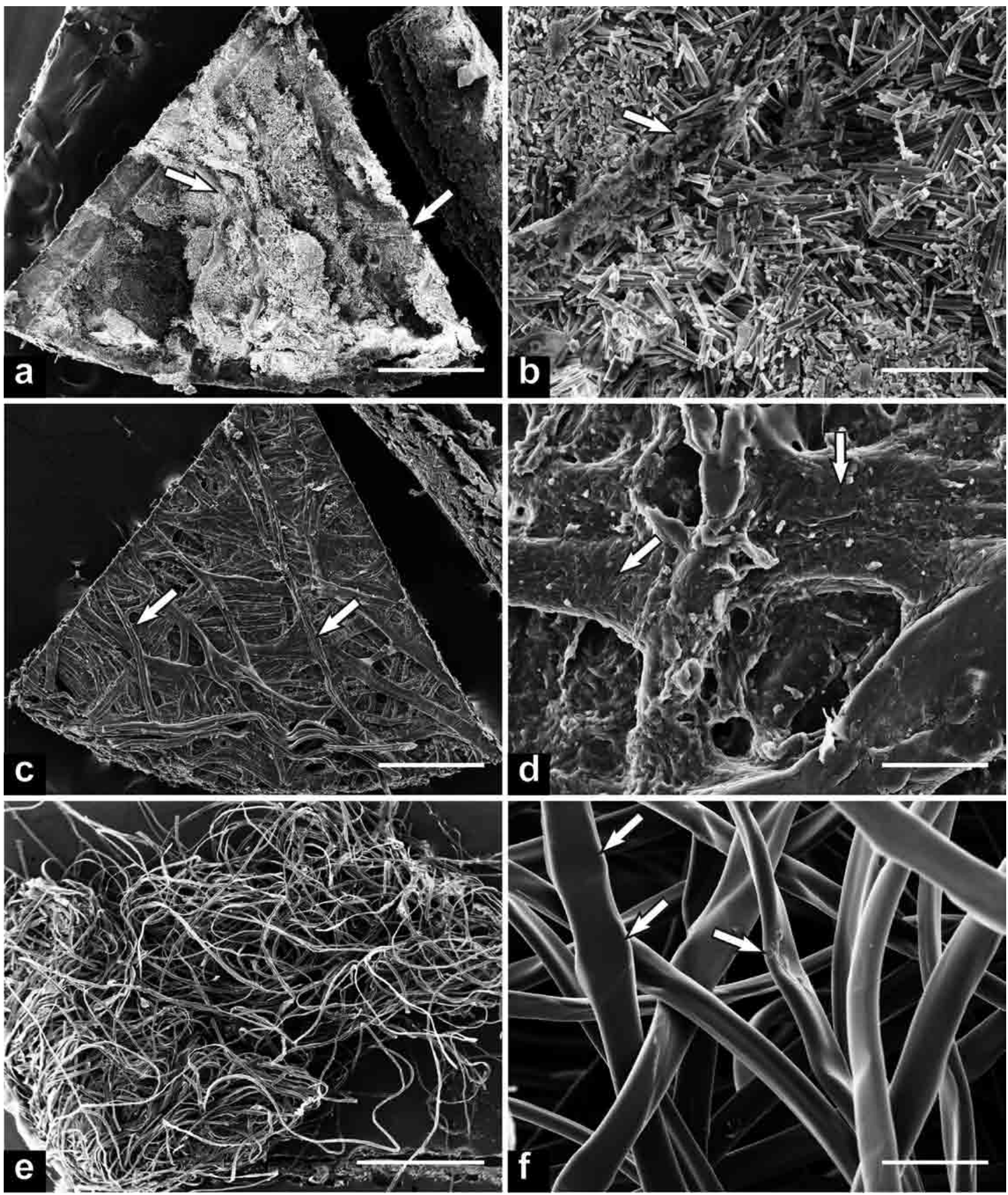

Figure 2. Low and high magnification SEM pictures of quadrants of cocoon discs of G. postica. Untreated (natural) cocoon disk, (a) low magnification (scale bar $500 \mu \mathrm{m}$ ) and (b) detail of covering mineral crystals (scale bar: $50 \mu \mathrm{m}$ ), with arrows indicating embedded fibers. Demineralized cocoon disk (c), with arrows indicating visible fibers in the cocoon shell network (scale bar: $500 \mu \mathrm{m}$ ), and (d) detail of cocoon disk surface showing fibers and gum, with arrows indicating crystal imprints (scale bar: $50 \mu \mathrm{m}$ ). A degummed cocoon disk, (e) low magnification (scale bar: $1 \mathrm{~mm}$ ), showing the unraveling of the disk, and (f) a detail of the fibers, with arrows indicating imprints showing that brins were deformed where they crossed one another in forming the network (scale bar: $50 \mu \mathrm{m}$ ).

cocoon (Figure 4c, 5 and 6). No evidence of remnants of the EDTA used for demineralization was found in the spectra of the demineralized cocoon discs (Figure 4c, 7).
We did not determine whether calcium oxalate was the only component removed by demineralization, which is likely not the case. 


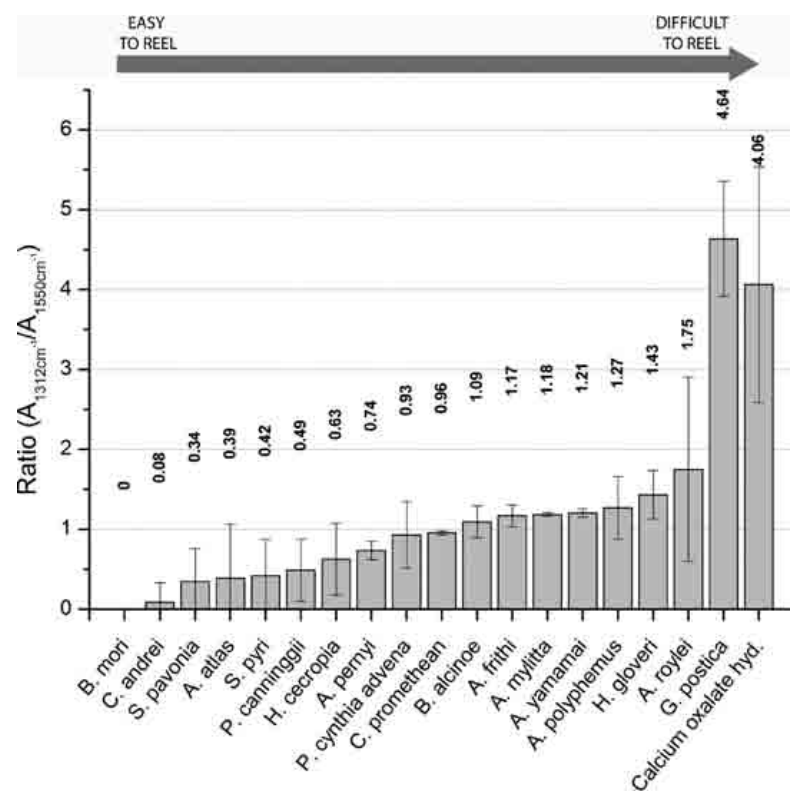

Figure 3. Ranking of the species according to the estimated approximated degree of mineralization obtained by the ratio of the FTIR spectra between the amide II peak (at $\sim 1590-1504 \mathrm{~cm}^{-1}$ ) and the calcium oxalate peak (at $\sim 1312 \mathrm{~cm}^{-1}$ ). The ease of reeling the cocoons according to this ranking is also shown. This graph shows that a cocoon shell like the one of B. mori that lacks a mineral coating is easy to reel, while the cocoon shell of G. postica being completely mineralized is difficult to reel with the currently used techniques.

Reeling. Prolonged degumming in a pineapple extract or in boiling sodium carbonate solution was effective in removing both the mineral and the gum, however the cocoons became impossible to reel because the fibers together with enclosed remains of the pupa produced tangles and finding the beginning of the fiber or reeling became impossible. The breakdown of the gum connections, separation of the fibroin brins, and their entanglement were seen in SEM images (Figure 2e,f), helping to explain why reeling was not possible after these treatments. In contrast, the five cocoons that were first demineralized were easy to reel (see Table 2). This is probably because the gum connections holding the fibers together were still intact preventing tangling yet sufficiently swollen and weakened to allow them to be broken by the forces generated by wet reeling. Additionally, removal of the mineral made it easy to find the beginning of the fibers. The total length of reeled silk varied from a minimum of $45 \mathrm{~m}$ to maximum of $572 \mathrm{~m}$ (Figure 5a). Although this is somewhat less than that which can be obtained from B. mori (300-1300 m; unpublished data), it still represents a commercially useful quantity. Continuous lengths up to $350 \mathrm{~m}$ (see Figure 5a: cocoon 1 and 4) could be reeled from G. postica cocoons, which represents a very great improvement over the short staple fibers produced by carding after degumming with pineapple extract. The much longer fibers should allow stronger and more even singles and twists to be made for the first time from this silk.

Almost all silk cocoons were reeled to completion and reeling stopped when only a thin transparent layer was left surrounding the pupa, as with B. mori reeled cocoons. The number of breaks varied but was comparable to the number in industrially reeled $B$. mori cocoons (see Table 2 ). The number of breaks in cocoon 5 was significantly higher than the other cocoons because the trap door of this cocoon was rectangular, snagging the fibers and causing them to break. To yield a large quantity of reeled silk, the G. postica cocoon needed a volume $>10 \mathrm{~mL}$ or more specifically, a width of $>20 \mathrm{~mm}$ and length $<22 \mathrm{~mm}$ (unpublished data).

The demineralizing method enabled G. postica cocoons to be reeled at a speed of $6 \mathrm{~m} / \mathrm{min}$ which is less than the 50 to $80 \mathrm{~m} / \mathrm{min}$ used industrially for multiend reeling of B. mori silk. However higher speeds were not tried for G. postica.

Cocoons from A. mylitta, A. pernyi, C. Andrei, S. canningii, and $G$. postica could be readily wet reeled after demineralizing as described above. Other species were not tried. Most cocoons available for this study had emergence holes where the larva broke through the cocoon shell. This led to breaking of the fibers at these spots, which in turn prevented the reeling of long continuous lengths of fiber. Accordingly, we have with a few exceptions no reliable data on maximal reelable length for most species. However, we do have reliable mechanical data on the effect of the treatments on fiber properties. Moreover, we also have reliable data on reeling length for one selected species, namely, G. postica.

Tensile Properties. Figure $5 \mathrm{~b}$ compares the stress strain curves for G. postica demineralized brins (black curves), demineralized/sodium carbonate degummed (red) brins, and brins from carded and spun commercial G. postica yarns that were derived from cocoons degummed with pineapple juice (blue). The first two treatments gave much more consistent pattern of stress strain curves probably as a result of less fiber damage produced by wet reeling compared with pineapple degumming and carding. Silk fibers reeled from demineralized cocoons were significantly $(p<0.001)$ and markedly stronger than the silk from commercial G. postica yarn as judged by their mean breaking load, $0.084 \mathrm{~N}$ against $0.058 \mathrm{~N}$, respectively (see Table 3). However, the mean breaking stress of the demineralized silk filaments was significantly less $(p<0.05)$ than that of the commercial yarn due to the large and highly significant $(p<0.001)$ reduction in the cross sectional area of the brins produced by the vigorous action of the pineapple extract. It is not clear whether the smaller mean cross sectional area of pineapple degummed material resulted solely from more efficient removal of gum or whether it additionally removed some weakly stabilized fibroin from the surface of the brin. Sodium carbonate degumming of previously demineralized fibers reduced the cross sectional area of the fiber resulting in an increased breaking stress (see Figure 5c,d). However, the break load did not significantly change $(p>0.05)$ after sodium carbonate degumming indicating that the gum did not contribute to the strength of the fiber.

Although the mean breaking energy of the demineralized/ degummed filament was not significantly greater $(p>0.05)$ than the pineapple degummed ones, they did show a significantly larger $(p<0.001)$ mean elongation to break (see Table 3$)$. Thus, the $G$. postica fibers produced by wet reeling after demineralizing and sodium carbonate degumming are more consistent in mechanical properties and significantly better in strength measured by breaking load and elongation to break compared with the commercial fibers from the same species produced by pineapple degumming, carding, and hand spinning. If a milder degumming method, comparable to the pineapple degumming in effectiveness of gum removal, were to be used in place of sodium carbonate degumming, it may result in a much smaller cross section of the demineralized fibers and improved breaking stress. Because the gum of the fiber does not contribute 

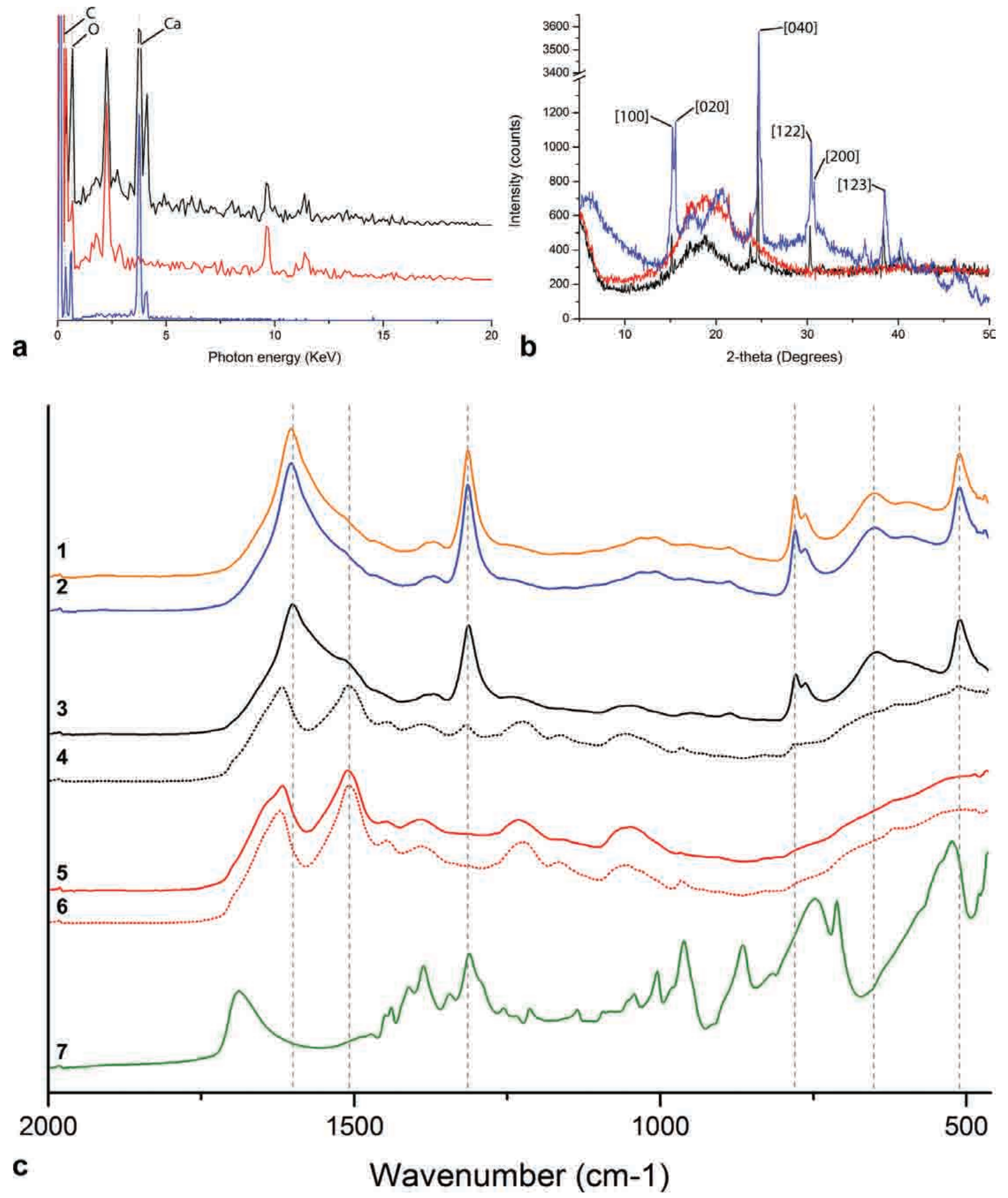

Figure 4. Spectra of the extracted mineral powder (blue trace), its presence in the natural cocoon shell (black trace) and its removal (red trace) from the cocoon shell by the demineralizing treatment (green trace). The EDX shows that the mineral powder consists of calcium, oxygen and carbon (a, blue trace), while the XRD spectra of the extracted powder (b, blue trace) of the G. postica cocoon is identified as, and indexed against whewellite (JCPDS card 01-077-1160). The EDX (a) and XRD (b) spectra both show that the peaks characteristic for calcium oxalate disappeared after the demineralization treatment (black line, natural cocoon disk; red line, demineralized cocoon disk). The FTIR spectra (c) showed that the extracted powder from the cocoons (2, blue line) matches the calcium oxalate hydrate spectra (1, orange line) bought from Sigma. The untreated (black line) cocoon discs (3) matched the calcium oxalate spectra (1, orange line) and that almost no mineral was present on the inside of the untreated disk (4). After demineralizing (red line), the calcium oxalate was completely removed on the outside (5) as well as on the inside (6) of the cocoon, with no traces of EDTA (7, green line).

to the breaking load of the fiber (Table 3A,B), it is possible to recalculate the breaking stress of a demineralized fiber based on the cross section of the pineapple degummed fibers (Table 3C). This means that our demineralized/degummed G. postica fibers 
compared favorably with a typical commercial degummed B. mori silk as follows: mean breaking stress $0.638,0.500 \mathrm{GPa}$; mean breaking strain 37.9, 15.2\%; initial modulus 8.3, 8.2 GPa; breaking energy $77.9,60 \mathrm{~J} \mathrm{~cm}^{-3}$ (see Table 3D,E).

\section{DISCUSSION}

It emerged from our studies that the cocoons of Wild Silkmoths belonging to a range of taxa can indeed be wet reeled and produce high-quality fibers using a demineralizing method novel for silkworm cocoons.

Table 1. Weight and Density of the G. postica Cocoon Discs of the Untreated Cocoons (A) versus the Effect of (B) Demineralization and Combined Demineralization and Sodium Carbonate Degumming (C) on the Weight and Density of the G. postica Cocoon Discs ${ }^{a}$

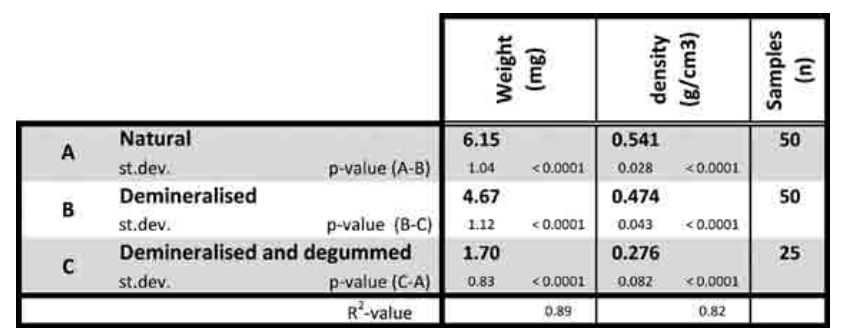

${ }^{a}$ Each box shows the mean, standard deviation and two tailed probability (ANOVA).
Using Gonometa postica cocoons as an example, we present strong evidence derived from SEM, EDX, FTIR and XRD demonstrating that calcium oxalate monohydrate (whewellite) forms a major structural component of the outer layer of the cocoon of $G$. postica and is present in smaller quantities throughout the rest of the cocoon shell. Our evidence also strongly suggests that the extensive infiltration of whewellite between the silk fibers accounts for the difficulty of reeling in this and many other Wild Silkworm species. We suggest that the currently used methods for softening Gonometa cocoons, namely prolonged retting or digestion with pineapple extract are effective in removing the mineral but simultaneously remove the gum that holds the fibers together resulting in a collapse of the cocoon's structure and entanglement of the silk fibers making reeling impossible. In contrast, removal of the mineral with EDTA left the gum intact, preventing collapse and entanglement of the cocoon fibers, but evidently softened them sufficiently to enable wet reeling for the first time for this species. This method that clearly differs from degumming can be referred to as "demineralizing". We show that substantially all of the silk in G. postica cocoons can be reeled in this way using standard commercial reeling equipment to produce a total length of silk of up to $570 \mathrm{~m}$ per cocoon in unbroken lengths of up to $350 \mathrm{~m}$. Crucially we show that the mechanical properties of the silk reeled in this way and subsequently degummed are markedly better than commercial silk fibers produced from the same batch of cocoons using pineapple juice degumming, carding, and hand-spinning. Equally

Table 2. Reeling Data of Five Different G. postica Cocoons ${ }^{a}$

\begin{tabular}{|c|c|c|c|c|c|c|c|c|c|}
\hline Cocoon & Cocoon before reeling & $\begin{array}{l}\text { Cocoon } \\
\text { height } \\
(\mathrm{mm})\end{array}$ & $\begin{array}{l}\text { Cocoon } \\
\text { width } \\
\text { (mm) }\end{array}$ & $\begin{array}{l}\text { Calculated } \\
\text { cocoon volume } \\
\text { (ml) }\end{array}$ & Reeled silk & $\begin{array}{c}\text { Fibre } \\
\text { breaks } \\
\text { (n) }\end{array}$ & $\begin{array}{c}\text { Reeled } \\
\text { length } \\
\text { (m) }\end{array}$ & Cocoon after reeling & $\begin{array}{c}\text { Reeling } \\
\text { completion }\end{array}$ \\
\hline 1 & & 50.08 & 21.36 & 12.0 & & 9 & 572.0 & & almost complete \\
\hline 2 & & 48.28 & 19.99 & 10.1 & & 7 & 434.2 & & almost complete \\
\hline 3 & & 43.15 & 18.62 & 7.8 & & 4 & 45.1 & & complete \\
\hline 4 & & 49.79 & 19.96 & 10.4 & & 3 & 332.2 & & complete \\
\hline 5 & & 48.12 & 22.34 & 12.6 & & 23 & 179.0 & & partial \\
\hline
\end{tabular}

${ }^{a}$ Measures of the cocoon (width, height, and volume), number of breaks during reeling, total length of silk reeled, and a visual estimate of the completeness of the reeling are tabulated for each cocoon. The pictures show the cocoon before and after reeling and the amount of reeled silk (white scale bar $=1 \mathrm{~cm})$. 

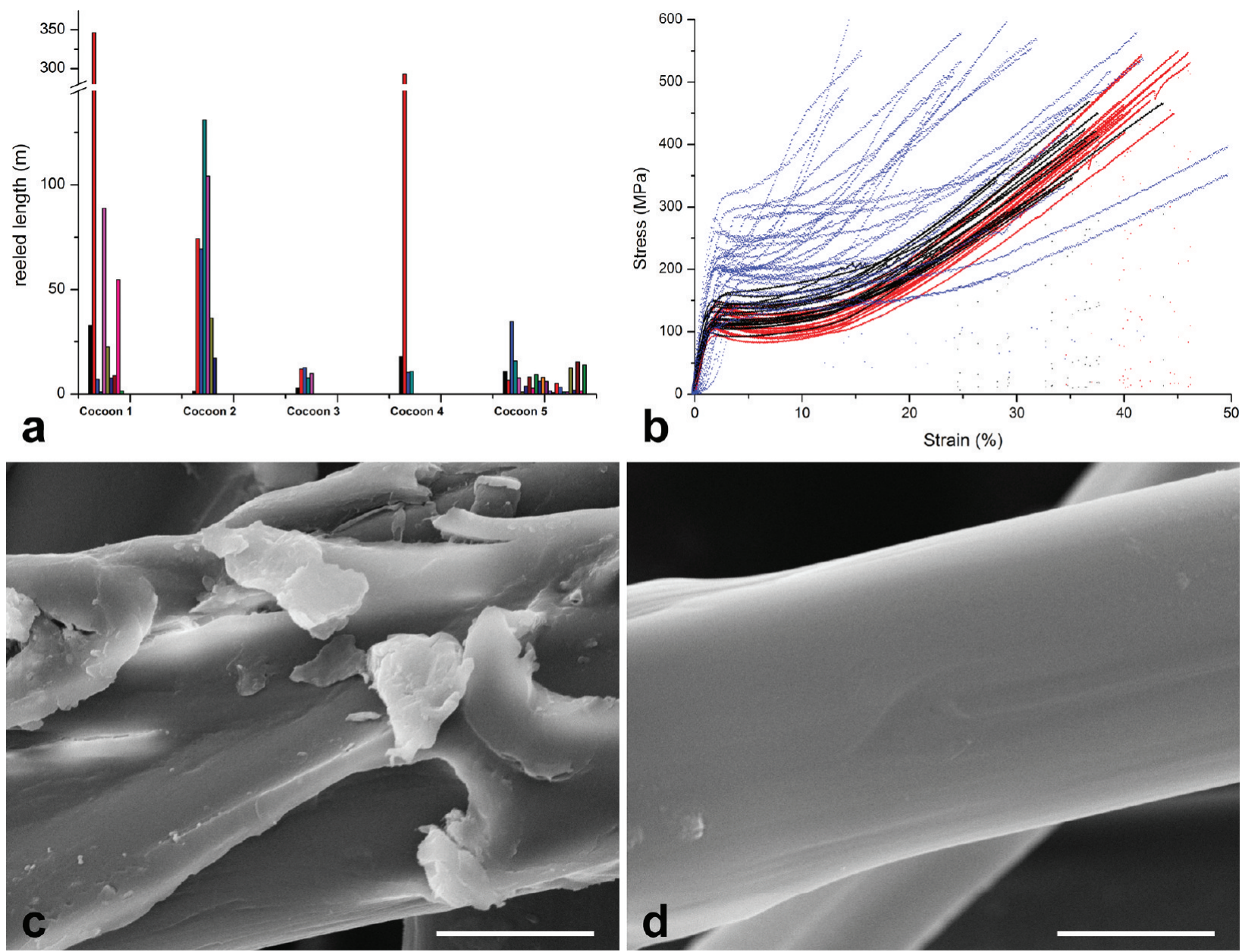

Figure 5. (a) Maximum continuous length of reeled silk fiber per cocoon, each column representing a new try after a fiber breakage. (b) Stress-strain graphs are shown from G. postica silk with different treatments: Treated with pineapple juice, carded, and unraveled from hand spun yarn (blue); Silk fibers extracted by reeling after demineralizing the cocoon before (black); and after sodium carbonate degumming (red). (c,d) SEM pictures of demineralized (c) and sodium carbonate degummed (d) fibers after reeling.

Table 3. Effect of Various Treatments on the Tensile Properties of G. postica Fibers ${ }^{a}$

\begin{tabular}{|c|c|c|c|c|c|c|c|c|}
\hline & & 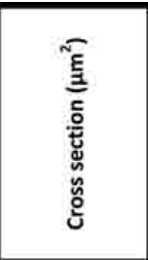 & 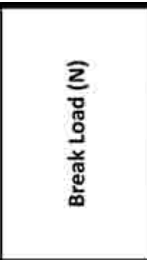 & 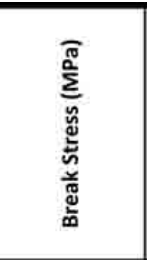 & 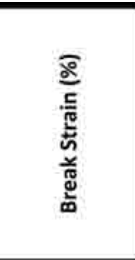 & 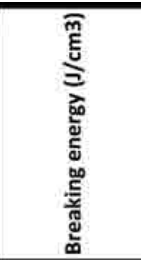 & 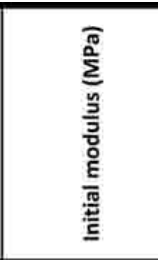 & 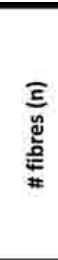 \\
\hline A & $\begin{array}{l}\text { Demineralised } \\
\text { st.dev. }\end{array}$ & $\begin{array}{cc}254 & \\
53 & <0.001\end{array}$ & $\begin{array}{cc}0.084 & \\
0.015 & 0.24\end{array}$ & $\begin{array}{|cc|}326 & \\
72 & <0.001\end{array}$ & $\begin{array}{cc}28.4 & \\
9.5 & <0.001\end{array}$ & $\begin{array}{cc}53.6 & \\
18.0 & <0.001\end{array}$ & $\begin{array}{c}8398 \\
1541\end{array}$ & 101 \\
\hline B & $\begin{array}{l}\text { Demineralised }+ \text { degummed } \\
\text { st.dev. } \\
\text { p-value }(B-C)\end{array}$ & $\begin{array}{|cc|}198 & \\
36 & <0.001\end{array}$ & \begin{tabular}{|c|c|}
0.082 & \\
0.016 & $<0.001$
\end{tabular} & $\begin{array}{cc}401 & \\
98 & 0.031\end{array}$ & $\begin{array}{c}37.9 \\
7.5\end{array}<0.001$ & $\begin{array}{cc}77.9 & \\
26.2 & 0.615\end{array}$ & $\begin{array}{l}8363 \\
1385\end{array}$ & 100 \\
\hline c & $\begin{array}{l}\text { Pineapple juice } \\
\text { st.dev. }\end{array}$ & $\begin{array}{|cc|}25 & \\
26 & <0.001 \\
\end{array}$ & $\begin{array}{|cc|}0.058 & \\
0.017 & <0.001\end{array}$ & $\begin{array}{cc}443 & \\
129 & <0.001\end{array}$ & $\begin{array}{cc}27.7 & \\
11.7 & 0.728\end{array}$ & $\begin{array}{cc}75.3 & \\
35.7 & <0.001\end{array}$ & $\begin{array}{cc}10960 & \\
3188 & <0.001\end{array}$ & 30 \\
\hline & $R^{2}$-value & 0.40 & 0.24 & 0.23 & 0.22 & 0.21 & 0.22 & \\
\hline & Re-calculated G. postica & $\begin{array}{c}125 \\
26\end{array}$ & $\begin{array}{c}0.080 \\
0.019\end{array}$ & $\begin{array}{l}638 \\
155\end{array}$ & $\begin{array}{c}37.9 \\
7.5\end{array}$ & 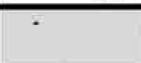 & $\overline{-}$ & 100 \\
\hline & $\begin{array}{l}\text { Commercially degummed } 8 . \text { mori } \\
\text { st.dev }\end{array}$ & $\underset{50}{280^{2}}$ & $\begin{array}{c}0.08^{1} \\
0.014\end{array}$ & $500^{2}$ & ${ }_{3.4}^{2}$ & $\begin{array}{c}65.1^{1} \\
19.3\end{array}$ & $8114^{1}$ & 10 \\
\hline
\end{tabular}

${ }^{a}$ (A) Demineralized and reeled; (B) demineralized, reeled, and sodium carbonate degummed; (C) fibers produced by degumming with pineapple juice, carded, hand spun, and unraveled. Also shown is a recalculation of the tensile properties demineralized, reeled, and sodium carbonate degummed G. postica fibers based on the cross section of pineapple degummed fibers (see text). Each box shows the mean (top left), standard deviation (bottom left), and two-tailed probability (bottom right, ANOVA). ${ }^{b}$ Juan Guan personal communication. ${ }^{c}$ Shao, Z. Z.; Vollrath, F. Surprising strength of silkworm silk. Nature 2002, 418 (6899), 741-741. 
crucially, when suitably degummed, the mechanical properties are also comparable to those of conventionally degummed commercial B. mori silk.

Importantly, other species of Wild Silkmoths with more established commercial potential such as Antheraea mylitta, Antheraea pernyi, Cricula andrei, and Samia canninggii responded to the EDTA treatment in a comparable fashion, suggesting that the treatment crosses not only species boundaries but also those of higher taxa (families) and, thus, has more generic implications.

\section{CONCLUSIONS}

We conclude that the demineralizing method described and explored here may provide a general method for softening most Wild Silk cocoons to enable them to be wet reeled. Importantly, the fibers of G. postica, which previously had not been examined in any great detail, when obtained this way showed competitive properties to fibers produced by the commercial mulberry silkworm B. mori. This suggests that our novel degumming method should increase the range of Wild Silkmoth species that can be wet reeled and may be important for the development of the silk industries not only in Asia but also in Africa and South America.

\section{AUTHOR INFORMATION}

\section{Corresponding Author}

*E-mail: tgheysens@googlemail.com; fritz.vollrath@zoo.ox.ac.uk.

\section{ACKNOWLEDGMENT}

For funding, we thank the AFOSR and EU for general support and the BBSRC and EPSRC for a CASE doctoral studentship to T.G. We thank Fujia Chen, Christopher Holland, Maxime Boulet-Audet, and David Porter for comments and suggestions on the research and preparation to this manuscript. We also thank Bjoern Greving for helping in demineralizing the cocoons.

\section{REFERENCES}

(1) Franck, R. R. In Silk, Mohair, Cashmere and Other Luxury Fibres, Woodhead Publ. and CRC Press: Cambridge and Boca Raton, FL, 2001; p 264.

(2) Das, S.; Chowdhury, S. K.; Das, N. K. J. Textile Inst. 1992, 83 (2), 279-281.

(3) Akai, H.; Nagashima, T. Int. J. Wild Silkmoth Silk 2003, 8, 1-5.

(4) Freddi, G.; Yoko Gotoh, Y.; Mori, T.; Tsutsui, I.; Tsukada, M. J. Appl. Polym. Sci. 1994, 52 (6), 775-781.

(5) Ohnishi, E.; Takahashi, S. Y.; Sonobe, H.; Hayashi, T. Science 1968, 160 (3829), 783-784.

(6) Shamitha, G.; Rao, A. P. Curr. Sci. 2006, 90 (12), 1667-1671.

(7) Brunet, P. C. J.; Coles, B. C. Proc. R. Soc. B 1974, 187 (1087), 133-170.

(8) Raven, D. J.; Earland, C.; Little, M. Biochim. Biophys. Acta 1971, 251 (1), 96.

(9) Das, S.; Chowdhury, S. K. J. Textile Inst. 1991, 82 (4), 505-507.

(10) Yolande, O. Master's thesis, Universiteit van die Vrystaat, Suid Afrika, 2007.

(11) Singh, L. R.; Devi, Y. R.; Devi, S. K. Electron. J. Biotechnol. 2003, 6 (3), 198-207.

(12) Veldtman, R. Ph.D. thesis, University of Pretoria, Hatfield, South Africa, 2005. 